\title{
A GRAVIDADE DO TRAUMA EM VÍTIMAS DE TRAUMATISMO CRÂNIO-ENCEFÁLICO AVALIADA PELO MANUAL AIS/90 E MAPAS CAIS/85
}

\author{
Regina Marcia Cardoso de Sousa* \\ Maria Sumie Koizumi** \\ Ana Maria Calil*** \\ Sonia Aurora A. Grossi**** \\ Lisbela Chaib*****
}

\begin{abstract}
SOUSA, R.M.C.de; KOIZUMI, M.S.; CALIL, A.M.; GROSSI, S.A.A.; CHAIB, L. A gravidade do trauma em vítimas de traumatismo crânico-encefálico avaliada pelo manual AIS/90 e mapas CAIS/85. Rev.latino-am.enfermagem, Ribeirão Preto, v. 6, n.1, p. 41-51, janeiro 1998.
\end{abstract}

Estudo comparativo do uso do manual da ABBREVIATED INJURY SCALE (AIS) e dos mapas da CONDENSED ABBREVIATED INJURY SCALE (CAIS), como bases para cálculo do INJURY SEVERITY SCORE (ISS) em vítimas de trauma crânio-encefálico. Os resultados evidenciaram que o valor do ISS foi coincidente na maioria (59,51\%) das vítimas passíveis de codificação pelos dois instrumentos. Quanto à indicação da faixa de gravidade do trauma (grave, moderado e leve) não existiram diferenças estatisticamente significantes entre os dois instrumentos. Quanto a capacidade de cobertura da CAIS/85 para a identificação da gravidade das lesões constatou-se que a CAIS/85 permitiu a pontuação de 61,38\% das lesões pontuadas com a AIS/90.

UNITERMOS: traumatismo crânio-encefálico, gravidade do trauma, avaliação

\section{INTRODUÇÃO}

Os acidentes e violências constituem um problema de graves proporções para a sociedade moderna pois são responsáveis por índices alarmantes de morbidade, mortalidade e incapacidades ${ }^{5,12,17,24}$.

A magnitude de suas conseqüências levaram pesquisadores e profissionais da área da saúde a criar índices de gravidade que permitam auxiliar a interpretação e avaliação das dimensões do trauma.

Índices de gravidade do trauma têm sido definidos como classificações numéricas vinculadas a características selecionadas do paciente, que proporcionam meios confiáveis e válidos para avaliar a probabilidade de mortalidade e morbidade após o trauma ${ }^{9}$.

Esses índices possibilitam a avaliação e comparação da assistência, bem como a efetividade das medidas preventivas instituídas. Podem também auxiliar no processo de triagem dos pacientes, no planejamento de serviços, na distribuição de recursos humanos e materiais, além de auxiliar em questões legais.

Dentre os índices de gravidade do trauma que têm sido desenvolvidos o INJURY SEVERITY SCORE (ISS), tem sido reconhecido como o mais promissor, por ser dotado de sensível poder prognóstico quanto à sobrevivência e mortalidade ${ }^{9}$. Além disso, há uma opinião freqüente que o ISS apresenta vantagens em relação a outros sistemas de avaliação da gravidade do trauma, uma vez que considera as lesões de diferentes regiões do corpo; baseia-se em diagnósticos de lesões confirmadas através de exames complementares, ou operações cirúrgicas ou inspeção direta; e especifica eventuais resultados do trauma.

O ISS é obtido a partir da aplicação da ABBREVIATED INJURY SCALE (AIS). A AIS é um sistema avaliativo, de base anatômica que classifica cada lesão decorrente de um trauma, por região corpórea e estabelece um escore que indica gravidade da lesão

\footnotetext{
* Professor Doutor do Departamento de Enfermagem Médico Cirúrgica da Escola de Enfermagem da Universidade de São Paulo ** Professor Titular do Departamento de Enfermagem Médico Cirúrgica da Escola de Enfermagem da Universidade de São Paulo *** Professor do Departamento de Enfermagem, Faculdades Integradas São Camilo

**** Professor Assistente do Departamento de Enfermagem Médico Cirúrgica da Escola de Enfermagem da Universidade de São Paulo

****** Professor do Departamento de Enfermagem Médico-Cirúrgica da Universidade Federal do Piaú
} 
específica. É apresentada numa escala ordinal de 6 pontos, oscilando de AIS 1 (lesão leve) a AIS 6 (lesão quase sempre fatal). Pela AIS obtém-se o escore de gravidade de uma lesão isoladamente.

O primeiro Manual para aplicação da AIS foi publicado em 1971, sendo a partir de então revisado periodicamente pela American Association for Automotive Medicine, com vista a seu refinamento. A AIS/71 continha 75 descrições de lesão enquanto que AIS $/ 90^{2}$ reune mais de 2.000 .

Tendo em vista a importância do conhecimento dos índices de gravidade do trauma dos pacientes, ainda durante a internação bem como a complexidade crescente do Manual AIS, dificultando seu uso rotineiro, diversos autores idealizaram instrumentos resumidos ou simplificado do Manual AIS.

Dentre esses instrumentos, o CONDENSED ABBREVIATED INJURY SCALE (CAIS), elaborado por CIVIL \& SCHWAB $^{8}$, é o mais desenvolvido. A primeira versão desse instrumento foi desenvolvida a partir do Manual AIS/80. Uma segunda versão foi elaborada reconhecendo as modificações propostas no AIS $/ 85^{1}$, apresentando-se assim, os Mapas CAIS $/ 85^{8}$ que foram anexados ao Manual desse mesmo ano ${ }^{1}$.

Os Mapas Condensados CAIS $/ 85^{8}$, oriundos do Manual AIS $/ 85^{1}$, versão mais atual dos mapas condensados que se tem acesso, é um instrumento de fácil e rápida aplicação, como bem demonstraram CIVIL \& $\mathrm{SCHWAB}^{8}$ que os idealizaram. No Brasil, DALOSSI \& $\mathrm{KOIZUMI}^{9}$, evidenciaram também tais características ao utilizá-los em vítimas de causas externas em geral.

O CAIS $/ 85^{8}$ é constituído por dois mapas: um para pontuar lesões de pacientes de trauma fechado CAIS/85F e outro para pontuar lesões de pacientes de trauma penetrante - CAIS/85P.

Os dois mapas têm forma de apresentação semelhante: mapa de página única, em que as descrições das lesões são apresentadas separadamente nas 6 regiões do corpo utilizadas para cálculo do ISS, e de acordo com os escores de gravidade AIS. Esses mapas incluem as lesões mais frequientemente verificadas nos pacientes de trauma.

Ainda que o Manual AIS em sua versão de $1990^{2}$, tenha se mostrado um eficiente instrumento para codificar e classificar lesões, apresentando importante avanço na codificação de lesões neurológicas, o volume de mais de 2.000 descrições de lesões dificultam seu uso corrente, particularmente quando se deseja pontuar o paciente numa Unidade de Emergência.

Considerando-se que, uma das partes mais ampliadas do Manual AIS, versão $1985^{1}$ para a versão $1990^{2}$ foi o segmento cabeça, a indagação quanto à efetividade do CAIS $/ 85^{8}$, nos pacientes admitidos nas Unidades de Emergência devido traumatismo crânioencefálico (TCE), permaneceu sem resposta.

São, pois, objetivos deste estudo:
- analisar comparativamente a gravidade do trauma de vítimas de TCE através do ISS resultante da aplicação do Manual AIS/902 e Mapas CAIS/ $/ 85^{8}$.

- verificar a capacidade de cobertura da CAIS $/ 85^{8}$ para identificar a gravidade das lesões descritas.

\section{MATERIAL E MÉTODOS}

A população estudada foi constituída por vítimas de TCE admitidas para tratamento no Instituto Central do Hospital das Clínicas da Faculdade de Medicina da Universidade de São Paulo no período de março a junho de 1993, cujo evento traumático ocorreu nesse mesmo período. Os pacientes deste estudo obedeceram aos seguintes critérios de inclusão: terem entre 12 e 60 anos de idade e terem sobrevivido ao evento traumático até a alta dessa instituição.

Considerando-se que todos os pacientes, após saída desse hospital, têm seu prontuário arquivado na Divisão de Arquivo Médico, a localização dos pacientes internados devido a TCE foi feita a partir de lista fornecida por essa divisão.

Nesta lista foram selecionados os pacientes sobreviventes, internados nos meses de março, abril, maio e junho de 1993, da faixa etária de 12 a 60 anos. Os pacientes reinternados em decorrência do trauma, só foram incluídos quando sua primeira internação também ocorreu no período especificado.

Feita esta seleção, iniciou-se o processo de busca de prontuário através do número do registro geral dos pacientes no hospital. Localizado o prontuário foram preenchidos os dados da ficha apresentada no Anexo 1.

$\mathrm{O}$ prontuário foi a fonte de identificação das lesões dos pacientes em estudo. As anotações registradas no prontuário, desde o momento da entrada do paciente até a alta, foram atentamente lidas. Com isso, as lesões decorrentes do trauma foram identificadas e transcritas para a lista de diagnósticos de lesão do Anexo 1.

Todas as lesões registradas no Anexo 1 foram analisadas para classificação de acordo com o Manual AIS $/ 90^{2}$ e Mapas CAIS $/ 85^{8}$ e quando pontuadas, consideradas para cálculo do ISS. Para isso, utilizou-se o Anexo 2 que foi preparado para permitir o cálculo do ISS tendo como base a classificação da gravidade das lesões estabelecidas por esses dois instrumentos.

Assim, após a pontuação das lesões dos pacientes pelo Manual AIS $/ 90^{2}$ realizou-se o cálculo do ISS. Da mesma forma procedeu-se em relação as pontuações obtidas pela CAIS $/ 85^{8}$.

O ISS é um índice matematicamente calculado que oferece o escore de gravidade do trauma de forma global. Para cálculo do ISS, é estabelecido o escore mais alto das lesões identificadas para cada uma das 6 regiões corporais: cabeça e pescoço; face; tórax; abdômen ou conteúdos pélvicos; membros ou cintura pélvica e 
superfície externa. Calcula-se então o ISS, que é resultado da soma dos quadrados dos escores AIS mais altos das três regiões diferentes do corpo mais gravemente traumatizadas ${ }^{2}$. Um exemplo apresentado no Anexo 3 ilustra a forma de aplicação desse índice neste estudo.

Os dados deste estudo foram processados eletronicamente e apresentados em freqüência absoluta e relativa sendo mostrados em forma de tabelas e figura.

Para complementar a análise dos dados obtidos fez-se necessário aplicar a Prova de associação pelo Quiquadrado, a qual foi feita admitindo um erro de primeira espécie de $5 \%{ }^{4}$.

\section{RESULTADOS E DISCUSSÃO}

\section{População estudada}

A população estudada constituiu-se de 213 vítimas de TCE (Tabela 1$)$ sendo $178(83,57 \%)$ do sexo masculino e $35(16,43 \%)$ do sexo feminino, numa proporção entre homens e mulheres de 5,08:1. As faixas etárias predominantes foram as de 12 à 23 anos e de 24 à 35 anos, incluindo respectivamente $69(32,39 \%)$ e 66 (30,99\%) vítimas, totalizando $135(63,38 \%)$ indivíduos com idade de até 35 anos.

\section{Tabela 1 - Distribuição das vítimas com TCE segundo idade e sexo. São Paulo, 1993}

\begin{tabular}{l|rrrr|rr}
\hline \multirow{2}{*}{ SEXO } & \multicolumn{2}{|c}{ MASCULINO } & \multicolumn{2}{c}{ FEMININO } & \multicolumn{2}{c}{ TOTAL } \\
IDADE & $\mathrm{N}^{\circ}$ & $\%$ & $\mathrm{~N}^{\circ}$ & $\%$ & $\mathrm{~N}^{\circ}$ & $\%$ \\
\hline $12-23$ & 58 & 27,23 & 11 & 5,16 & 69 & 32,39 \\
2435 & 57 & 26,76 & 9 & 4,23 & 66 & 30,99 \\
$36-47$ & 42 & 19,72 & 12 & 5,63 & 54 & 25,35 \\
$48-60$ & 21 & 9,86 & 3 & 1,41 & 24 & 11,27 \\
\hline TOTAL & 178 & 83,57 & 35 & 16,43 & 213 & 100,00 \\
\hline
\end{tabular}

No geral, a distribuição dos participantes do estudo quanto à idade apresentou características semelhantes a de estudos em populações de traumatizados: o grupo mais jovem (12 a 23 anos) foi o mais presente e a frequiência das vítimas diminuiu à medida que a idade aumentou.

Várias publicações indicam o adulto jovem do sexo masculino como a mais freqüente vítima de $\mathrm{TCE}^{3,6,13}$. A predominância do sexo masculino tem estado presente em todos estudos analisados relacionados a trauma em geral e TCE, havendo porém diferenças bastante acentuadas nas proporções estabelecidas. Entre as publicações referentes a trauma em geral, destacamse os dados do Major Trauma Outcome Study ${ }^{7}$ que apresenta 57.231 homens e 22.599 mulheres, perfazendo proporção de 2,5:1. Referente a TCE especificamente KRAUS $^{17}$ observou na revisão de 9 estudos americanos, que em 8 era mostrado uma incidência de 2 ou mais homens comparados com mulheres. MASET et al. ${ }^{21}$ verificaram uma incidência de 2,37 homens para 1 mulher. Dados do Banco Internacional apresentados por JENNETT et al. ${ }^{14,15}$ com 1.000 vítimas de TCE grave, mostram uma predominância de homens de 5:1. Proporção ainda mais alta foi encontrada no estudo de SILVA et al. ${ }^{23}$ de seguimento de pacientes com TCE grave em Londrina, onde $91 \%$ das vítimas eram do sexo masculino, perfazendo uma proporção de 10:1.

Analisando a incidência de TCE por idade, em publicações americanas, KRAUS ${ }^{17}$ verificou que houve um pico nos jovens com idade entre 15 e 24 anos. Outros autores $^{6,12}$ ampliam essa faixa etária, quando indicam a maior incidência de TCE entre 15 e 29 anos e MASET et $\mathrm{al}^{21}$, analisando as ocorrências de TCE na região de São José do Rio Preto, apontam a mais alta incidência entre 20 e 29 anos.

Quanto às demais faixas etárias, KRAUS ${ }^{17}$ mostrou que há curva descendente de incidência de TCE após 15 e 24 anos, com um segundo pico ocorrendo em idosos (maiores de 60 anos). No estudo de MASET et al. $^{21}$, após a faixa etária de 20 a 29 anos, a incidência declina. Entretanto, entre 50 e 59 anos ela se eleva levemente e mantém-se no mesmo nível entre os mais velhos.

Tabela 2 - Distribuição das vítimas de TCE segundo idade e causa externa. São Paulo, 1993

\begin{tabular}{|c|c|c|c|c|c|c|c|c|c|c|}
\hline \multirow{2}{*}{$\begin{array}{l}\text { IDADE } \\
\text { CAUSA EXTERHA }\end{array}$} & \multicolumn{2}{|c|}{$12-23$} & \multicolumn{2}{|c|}{2435} & \multicolumn{2}{|c|}{$36-47$} & \multicolumn{2}{|c|}{$48-60$} & \multicolumn{2}{|c|}{ TOTAL } \\
\hline & $\mathrm{N}^{\circ}$ & $\%$ & $\mathrm{~N}^{\circ}$ & $\%$ & $\mathrm{~N}^{\circ}$ & $\%$ & $\mathrm{~N}^{\circ}$ & $\%$ & $\mathrm{~N}^{\circ}$ & $\%$ \\
\hline veículo à motor & 38 & 17,84 & 31 & 14,55 & 22 & 10,33 & 7 & 3,29 & 98 & 46,01 \\
\hline queda acidental & 22 & 10,33 & 23 & 10,80 & 26 & 12,20 & 13 & 6,10 & 84 & 39,43 \\
\hline agressão & 4 & 1,88 & 1 & 0,47 & - & - & 1 & 0,47 & 6 & 2,82 \\
\hline demais acidentes* & 5 & 2,35 & 11 & 5,16 & 6 & 2,82 & 3 & 1,41 & 25 & 11,74 \\
\hline TOTAL & 69 & 32,40 & 66 & 30,98 & 54 & 25,35 & 24 & 11,27 & 213 & 100,00 \\
\hline
\end{tabular}

* Inclui acidente não especificado; acidente de trânsito com outro tipo de veículo; lesão a qual se ignora se foi acidental ou intencionalmente infligida. 
Na Tabela 2, observa-se que os acidentes de trânsito de veículo a motor e as quedas acidentais foram as causas externas que mais frequientemente ocasionaram o TCE. O predomínio dos acidentes de trânsito, em relação as outras causas externas ocorreu nas faixas etárias até 35 anos. Entre os mais velhos, a mais freqüente ocorrência foram as quedas acidentais.

Segundo GARDNER ${ }^{12}$, é estimado que nos Estados Unidos, $49 \%$ dos casos de TCE, resultam de acidente de veículo a motor sendo seguido por quedas, acidentes esportivos e violência. No Brasil, de uma forma global naqueles que morrem devido causas externas, os acidentes de trânsito situam-se em primeiro lugar, seguido pelo homicídio ${ }^{5}$.

Nos participantes deste estudo, o tipo de trauma esteve relacionado com a idade das vítimas. Várias publicações têm indicado que o tipo do trauma é dependente da idade: entre adolescentes e adultos jovens predominam os acidentes de veículos a motor, enquanto em pessoa mais velhas são mais freqüentes as quedas, os atropelamentos e os assaltos ${ }^{16,19,20,22,24,25}$.

\section{Comparação dos resultados do ISS com a aplicação do Manual AIS/90 ${ }^{2}$ e Mapas CAIS/85 ${ }^{8}$}

O primeiro fato que chamou a atenção ao pontuar a gravidade das lesões das 213 vítimas que atenderam aos critérios de inclusão deste estudo, foi que todas puderam ser pontuadas através do Manual AIS $/ 90^{2}$, porém, ao aplicar os Mapas CAIS/ $85^{8}, 8$ (3,76\%) vítimas não foram pontuadas. A mais freqüente limitação na pontuação de descrições de lesões, foi a impossibilidade da CAIS $/ 85^{8}$ valorar o escore da Escala de Coma de Glasgow (ECGl) na admissão das vítimas, dado este passível de codificação se utilizada a seção nível de consciência da AIS $/ 90^{2}$.

Tabela 3 - Distribuição das vítimas de TCE segundo a relação entre os escores de gravidade do trauma, obtidos pela AIS/90 ${ }^{2}$ e CAIS/85 ${ }^{8}$. São Paulo, 1993

\begin{tabular}{l|cc}
\hline ESCORES DE GRAVIDADE & N & $\%$ \\
DO TRAUMA & & \\
\hline ISS $/ A I S / 90^{2}=$ ISS/CAIS $/ 85^{8}$ & 122 & 59,51 \\
${\text { ISS } / A I S / 90^{2} \text { ISS/CAIS } / 85^{8}}^{2}$ & 75 & 36,59 \\
ISS $/ A I S / 90^{2}<$ ISS/CAIS $/ 85^{8}$ & 8 & 3,90 \\
\hline TOTAL & $205^{*}$ & 100,00 \\
\hline
\end{tabular}

* Exclui 8 vítimas com descrição de lesões não passíveis de codificação pelo CAIS $/ 85^{8}$.

A Tabela 3 apresenta a relação entre os escores de gravidade do trauma (ISS) segundo obtenção por meio da AIS $/ 90^{2}$ e CAIS $/ 85^{8}$ e evidencia que das 205 vítimas passíveis de codificação pelos 2 instrumentos, 122 $(59,51 \%)$ apresentaram ISS coincidente e $83(40,49 \%)$ não coincidente. Destas, $75(36,59 \%)$ tiveram o ISS maior e $8(3,90 \%)$ o ISS menor pelo AIS $/ 90^{2}$ em relação à CAIS/ $85^{8}$.

Dos 122 (100\%) casos de ISS coincidentes, 101 $(82,64 \%)$ obtiveram idênticas pontuações nas lesões apresentadas e $21(17,36 \%)$ ainda que com mesmo escore de gravidade do trauma (ISS), obtiveram pontuações diferentes em lesões diagnosticadas (AIS).

A grande maioria dos $83(75+8)$ casos não coincidentes, obtiveram pontuações diferentes devido a menor abrangência na possibilidade de pontuação das lesões e menor valoração pela CAIS $/ 85^{8}$ em relação à AIS $/ 90^{2}$, principalmente no segmento corpóreo cabeça e pescoço. Isto fica evidente quando se observa que a CAIS/ $85^{8}$ não permite a codificação de diagnósticos de lesão como "brain swelling", pneumoencéfalo, lesão axonal difusa, fístula liquórica bem como, não permite a utilização direta do índice fisiológico ECGl possível de codificação pela AIS $/ 90^{2}$. Outro fato que se mostrou relevante na pontuação da gravidade do trauma no segmento corpóreo cabeça e pescoço foi a maior pontuação atribuída aos hematomas intracranianos pela AIS $/ 90^{2}$ em relação à CAIS $/ 85^{8}$, que ocorreu quando os descritores dessa lesão incluíram adjetivos como grande, extenso e massivo.

$\mathrm{Na}$ lista de lesões da AIS $/ 90^{2}$ foram incluídos vários sinônimos e descrições semelhantes, para permitir codificar apropriadamente descrições equivalentes de lesões que são encontradas nos prontuários dos pacientes. $\mathrm{O}$ aperfeiçoamento deste instrumento, além de aumentar sua confiabilidade, entre e inter codificadores, ainda permitiu que muitas lesões fossem melhor avaliadas a partir dos registros dos prontuários.

Através dos índices de gravidade do trauma as vítimas têm sido categorizadas em três grupos a saber: aquelas com trauma leve (ISS $\leq 15$ ), moderado (ISS entre 16 e 24 ) e grave (ISS $\geq 25$ ). O valor do ISS maior ou igual à 16 é considerado como ponto crítico, indicando assim trauma importante . $^{9,26}$.

Tabela 4 - Distribuição das vítimas de TCE segundo categoria de gravidade do trauma e os instrumentos utilizados (AIS/90 ${ }^{2}$ e CAIS/85 ${ }^{8}$ ). São Paulo, 1993

\begin{tabular}{l|cccc}
\hline INSTRUMENTO & \multicolumn{2}{|c}{${\text { AIS } / 90^{2}}^{2}$} & \multicolumn{2}{c}{ CAIS $/ 85^{8}$} \\
GRAVIDADE DO TRAUMA & $\mathrm{N}^{\circ}$ & $\%$ & $\mathrm{~N}^{\circ}$ & $\%$ \\
\hline leve (ISS $\leq 15)$ & 120 & 56,34 & 118 & 57,56 \\
moderado (ISS 16-24) & 65 & 30,52 & 71 & 36,64 \\
grave (ISS $\geq 25$ ) & 28 & 13,14 & 16 & 7,80 \\
\hline TOTAL & 213 & 100,00 & $205^{*}$ & 100,00 \\
\hline * Exclui 8 vítimas com descrição de lesões não passíveis de \\
codificação pelo CAIS $/ 85^{8}$.
\end{tabular}

$$
\begin{aligned}
& \mathrm{X}_{\mathrm{OBS}}^{2}=3,40 \\
& \mathrm{gl}=2
\end{aligned}
$$


A distribuição das vítimas segundo a gravidade do trauma (ISS) mensurada pela AIS $/ 90^{2}$ e CAIS $/ 85^{8}$ pode ser visualizada através da Tabela 4 que mostra que a maioria das vítimas pontuadas tanto pela $\mathrm{AIS} / 90^{2}$ como pela CAIS $/ 85^{8}$ concentram-se na menor faixa de gravidade do trauma (ISS $\leq 15$ ) respectivamente 120 $(56,34 \%)$ e $118(57,56 \%)$. Um número expressivo de vítimas foi encontrado na faixa de gravidade moderada (entre 16 e 24$)$ sendo $65(30,52 \%)$ pela AIS $/ 90^{2}$ e 71 $(36,64 \%)$ pela CAIS $/ 85^{8}$.

Para verificar se houve diferença estatisticamente significante na categorização da gravidade do trauma (ISS) quando pontuada pelo Manual AIS $/ 90^{2}$ e Mapas CAIS $/ 85^{8}$ foi aplicado o teste Qui-quadrado admitindose um erro de primeira espécie de $5 \%$. Ainda que diferenças proporcionais existam, a diferença observada não foi estatisticamente significativa, indicando que a distribuição das vítimas nos diferentes níveis de gravidade foi semelhante quando se utilizou esses dois instrumentos para categorização de gravidade do trauma.

Além disso, tendo como base o ponto crítico de gravidade do trauma o escore $\geq 16$, observa-se através da análise da Tabela 4 que 43,66\% $(30,52 \%+13,14 \%)$ das vítimas tiveram indicação de trauma importante através do Manual AIS $/ 90^{2}$ e 42,44\% (34,64\% + 7,80\%) pelos Mapas CAIS $/ 85^{8}$, estabelecendo uma discordância para essa indicação de apenas 1,22\% quando utiliza-se esses dois instrumentos.

Tal resultado é de expressiva importância para a indicação do uso da CAIS $/ 85^{8}$ para cálculo da gravidade do trauma (ISS), em serviços de emergência, a medida que tem se destacado o potencial deste índice na decisão de transferir o paciente de um hospital para outro. Tem sido recomendado por vários pesquisadores que pacientes com ISS $\geq 16$ sejam tratados em centros de trauma, uma vez que necessitam de sofisticados recursos diagnósticos e pessoal especializado ${ }^{10,11,18}$.

Tabela 5 - Distribuição das vítimas de TCE segundo valores obtidos no ISS quando pontuado pela CAIS/85 ${ }^{8}$ e AIS/90². São Paulo, 1993

\begin{tabular}{l|cccccc|cc}
\hline & \multicolumn{2}{|c}{$\leq 15$} & \multicolumn{2}{c}{$16-24$} & \multicolumn{2}{c}{$\geq 25$} & \multicolumn{2}{c}{ TOTAL } \\
${\text { ISS/CAIS } / 85^{8}}_{\text {ISS/AIS } / 90^{2}}$ & $\mathrm{~N}^{\circ}$ & $\%$ & $\mathrm{~N}^{\circ}$ & $\%$ & $\mathrm{~N}^{\circ}$ & $\%$ & $\mathrm{~N}^{\circ}$ & $\%$ \\
\hline$\leq 15$ & $\mathbf{1 1 0}$ & 53,66 & 3 & 1,46 & - & - & 113 & 55,12 \\
$16-24$ & 4 & 1,95 & $\mathbf{6 1}$ & 29,76 & - & - & 65 & 31,71 \\
$\geq 25$ & 4 & 1,95 & 7 & 3,41 & $\mathbf{1 6}$ & 7,81 & 27 & 13,17 \\
\hline TOTAL & 118 & 57,56 & 71 & 34,63 & 16 & 7,81 & $205 *$ & 100,00 \\
\hline
\end{tabular}

* Exclui 8 vítimas com descrição de lesões não passíveis de codificação pelo CAIS/85 ${ }^{8}$

A Tabela 5 evidencia uma alta concordância nas vítimas pontuadas pelos 2 instrumentos, ou seja, concordância de $110(53,66 \%)$ na faixa de gravidade $\leq$ 15 , de $61(29,76 \%)$ na faixa entre $16-24$ e de 16 $(7,81 \%)$ na faixa $\geq 25$, perfazendo um total expressivo de 187 (91,23\%). Entre 18 vítimas (8,77\%) não houve concordância nas faixas de gravidade indicada pelos dois instrumentos. Esta discordância foi mais acentuada entre as vítimas que tiveram indicação de trauma grave quando pontuadas pela $\mathrm{AIS} / 90^{2}$ (ISS $\geq 25$ ), sendo 11 $(4+7)$ o número de vítimas nesta condição . A partir desta observação retomou-se os dados da Tabela 4, e aplicou-se o teste Qui-quadrado considerando as vítimas com ISS $<25$ e aquelas com ISS $\geq 25$. Este teste foi aplicado para identificar eventuais diferenças significativas na indicação de trauma grave quando utiliza-se a AIS $/ 90^{2}$ e a CAIS $/ 85^{8}$ para pontuar as lesões. Os resultados mostraram mais uma vez, não haver diferença estatisticamente significante. Os dados das Tabelas 4 e 5 indicam portanto que quando as vítimas sobreviventes de TCE apresentam descrição de lesões passíveis de codificação pelos dois instrumentos, a categorização de gravidade do trauma (ISS) obtida é equivalente.

Para verificar a capacidade de cobertura da CAIS/ $85^{8}$ para identificar a gravidade de lesões descritas no prontuário, comparou-se o número de descrições de lesões pontuadas pela CAIS $/ 85^{8}$ com o número pontuado com o uso do Manual AIS $/ 90^{2}$.

Das $901(100,00 \%)$ descrições de lesões classificadas pelo Manual AIS $/ 90^{2}, 553$ (61,38\%) puderam também ser pontuadas pela CAIS $/ 85^{8}$. Contudo, como pode-se observar na Figura 1, a não pontuação de lesões identificadas, por não estarem listadas na CAIS/ $85^{8}$, ocorreu de forma diferenciada conforme a região corpórea. 
Figura 1 - Comparação do número de descrições de lesões pontuadas através dos instrumentos AIS/90² e CAIS $/ \mathbf{8 5}^{8}$, segundo região corpórea. São Paulo, 1993

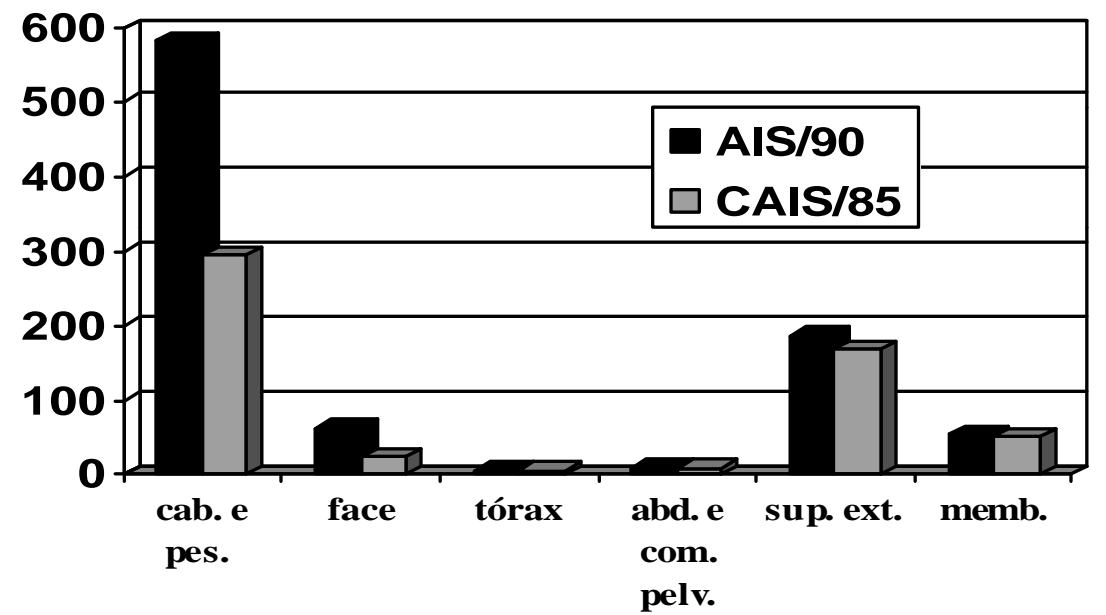

A Figura 1, demostra coerência na distribuição de lesões pontuadas com o perfil da população estudada através dos dois instrumentos, quando apresenta maioria de lesões na cabeça e pescoço. Entretanto, o número de descrições de lesões pontuadas no segmento corpóreo cabeça e pescoço, através do Manual AIS $/ 90^{2}$ foi aproximadamente 2 vezes maior que os pontuados através dos Mapas CAIS $/ 85^{8}$.

Destaca-se ainda uma diferença expressiva no número de descrições de lesões pontuadas no segmento corpóreo face, entre os dois instrumentos. O número de lesões pontuadas neste segmento corpóreo foi aproximadamente 3 vezes maior através da AIS $/ 90^{2}$.
Estes resultados confirmam as afirmações feitas, no próprio Manual AIS $/ 90^{2}$, sobre a maior abrangência do mesmo na pontuação de lesões cerebrais, e evidenciam a maior possibilidade de pontuação das lesões de face com esse instrumento. Nos outros segmentos corpóreos houve equivalência no número de lesões pontuadas pela $\mathrm{AIS} / 90^{2}$ e CAIS $/ 85^{8}$.

A confirmação da maior abrangência do Manual AIS $/ 90^{2}$ na pontuação das lesões no segmento corpóreo cabeça/pescoço, devido a substancial ampliação da AIS/ 90 em relação às lesões cerebrais, apontou a necessidade de realização de uma análise específica deste segmento corpóreo através do escore mais alto para a região cabeça.

Tabela 6 - Distribuição das vítimas de TCE segundo escore mais alto para região cabeça pontuada pela AIS/90 ${ }^{2}$ (M/AIS/90) e CAIS/85 ${ }^{8}$ (M/CAIS/85). São Paulo, 1993

\begin{tabular}{|c|c|c|c|c|c|c|c|c|c|c|c|c|}
\hline \multirow{2}{*}{$\begin{array}{l}\text { M/CAIS } / 85^{8} \\
\text { M/AIS/ } 90^{2}\end{array}$} & \multicolumn{2}{|c|}{1} & \multicolumn{2}{|c|}{2} & \multicolumn{2}{|c|}{3} & \multicolumn{2}{|c|}{4} & \multicolumn{2}{|c|}{5} & \multicolumn{2}{|c|}{ TOTAL } \\
\hline & $\mathrm{N}^{\circ}$ & $\%$ & $\mathrm{~N}^{\circ}$ & $\%$ & $\mathrm{~N}^{\circ}$ & $\%$ & $\mathrm{~N}^{\circ}$ & $\%$ & $\mathrm{~N}^{\circ}$ & $\%$ & $\mathrm{~N}^{\circ}$ & $\%$ \\
\hline 1 & 4 & 2,29 & 1 & 0,57 & - & - & - & - & - & - & 5 & 2,86 \\
\hline 2 & 2 & 1,14 & 35 & 20,00 & - & - & - & - & - & - & 37 & 21,14 \\
\hline 3 & 1 & 0,57 & 7 & 4,00 & 40 & 22,86 & 3 & 1,71 & - & - & 51 & 29,14 \\
\hline 4 & - & - & - & - & 1 & 0,57 & 62 & 35,43 & - & - & 63 & 36,00 \\
\hline 5 & - & - & - & - & - & - & 7 & 4,00 & 12 & 6,86 & 19 & 10,86 \\
\hline TOTAL & 7 & 4,00 & 43 & 24,57 & 41 & 23,43 & 72 & 41,14 & 12 & 6,86 & $175^{*}$ & 100,00 \\
\hline
\end{tabular}

* Exclui 38 vítimas com descrição de lesões na região cabeça não passíveis de codificação através do CAIS/858.

Das 213 vítimas sobreviventes de TCE, 38 $(17,84 \%)$ delas apresentaram descrição de lesão na região da cabeça que não foram passíveis de codificação pela CAIS $/ 85^{8}$. Do total das 175 vítimas com lesões pontuadas na região cabeça/pescoço através dos dois instrumentos, $153(87,43 \%)$ apresentaram o escore mais alto para região cabeça coincidente. Dos $22(12,57 \%)$ casos não coincidentes $18(10,88 \%)$ receberam pontuações maiores e $4(2,28 \%)$ receberam pontuações menores, quando o
Manual AIS $/ 90^{2}$ foi utilizado.

A não coincidência na maior pontuação da região cabeça $(n=22)$ foi mais freqüente nas vítimas com escore $3(n=11)$ e escore $5(n=7)$ quando se utiliza como referência a AIS $/ 90^{2}$. Isto deve-se à duas modificações apresentadas no Manual AIS $/ 90^{2}$ : este instrumento permite codificar fraturas de base de crânio através de sinais clínicos e a seção referente às lesões cerebrais foi substancialmente modificada nos seus escores 
principalmente no que se refere à contusões e hematomas. As contusões cerebrais nesse instrumento podem variar de escore AIS 3 à 5 de acordo com seu tamanho, localização e multiplicidade. Os hematomas cerebrais e cerebelares, tiveram sua pontuação revisada para refletir de forma mais adequada a gravidade do TCE.

Referente aos dados da Tabela 6 faz-se necessário ressaltar que a impossibilidade de pontuação de descrições de lesões na cabeça em 38 vítimas, através da CAIS $/ 85^{8}$, representa um percentual expressivo $(17,84 \%)$, sugerindo cautela na utilização deste instrumento na avaliação de vítimas de TCE. Contudo na categorização da gravidade do trauma em geral, os dois instrumentos mostraram equivalência pelo que é possível concluir que os Mapas condensados, CAIS $/ 85^{\circ}$, além de sua praticidade, oferecem segurança de aplicação na avaliação de vítimas nos serviços de emergência.

Finalmente pode-se afirmar que a gravidade das vítimas de trauma em geral pode ser agilizada nos serviços de emergência com a utilização da CAIS $/ 85^{8}$, mesmo após a revisão realizada no ano de $1990^{2}$ ter incorporado melhorias importantes na pontuação e descrições de lesões.

\section{CONCLUSÕES}

$\mathrm{Na}$ análise comparativa da gravidade das vítimas de TCE indicada pelo ISS resultante da pontuação pelo Manual AIS $/ 90^{2}$ e Mapas CAIS $/ 85^{8}$ verificou-se que:

- o valor do ISS foi coincidente na maioria $(59,51 \%)$ das vítimas passíveis de codificação pelos 2 instrumentos, sendo as diferenças resultantes na grande maioria (em 75 de 83 vítimas) devido a menor abrangência na possibilidade de pontuação das lesões e escore mais baixo pelo CAIS $/ 85^{8}$ em relação à $\mathrm{AIS} / 90^{2}$;

- quanto a indicação de trauma leve, moderado e grave, observou-se que não houve diferença estatisticamente significante quando se utilizou o Manual AIS $/ 90^{2}$ e Mapas CAIS $/ 85^{8}$, mesmo na indicação de trauma grave, faixa de gravidade em que as discordâncias foram mais freqüentes. Além disso, a discordância de indicação de trauma importante ( ISS $\geq 16$ ) foi de apenas $1,22 \%$.

Quanto a capacidade de cobertura da CAIS $/ 85^{8}$ para se identificar a gravidade de lesões descritas no prontuário constatou-se que:

- a CAIS $/ 85^{8}$ permitiu a pontuação de $61,38 \%$ das lesões pontuadas com a AIS $/ 90^{2}$ e as regiões corpóreas mais afetadas com a não pontuação foram: cabeça/pescoço e face;

- das 213 vítimas sobreviventes de TCE analisadas, 38 $(17,84 \%)$ não apresentaram descrição de lesão na região da cabeça passíveis de pontuação pela CAIS $/ 85^{8}$, porém, das 175 vítimas pontuadas na região da cabeça através dos 2 instrumentos, $153(87,43 \%)$ apresentaram escore mais alto na região cabeça coincidente.

\section{INJURY SEVERITY MEASURES BY AIS/90 MANUAL AND CAIS/85 CHART IN HEAD INJURED PATIENTS}

This study was developed in order to compare the use of the ABBREVIATED INJURY SCALE (AIS) and the CONDENSED ABBREVIATED INJURY SCALE (CAIS) as basis to calculate INJURY SEVERITY SCORE (ISS) in head injured patients. The results showed that the ISS value was equivalent in the majority of the patients (58,51\%) codified by both scales. Also no statistic differences between the scales were perceived when we compared the severity levels as severe, moderate and minor. 61,38\% of the lesions scored by AIS/90 were scored by CAIS/85, too.

\section{KEY WORDS: brain injury, injury severity, assessment}

\section{LA GRAVEDAD DEL TRAUMA EN VÍCTIMAS DE TRAUMATISMO CRÁNEO- ENCEFÁLICO POR MEDIO DEL MANUAL AIS/90 Y MAPAS CAIS/85}

Estudio comparativo del uso del Manual de la ABBREVIATED INJURY SCALE (AIS) y de los mapas de la CONDENSED ABBREVIATED INJURY SCALE (CAIS), como base para el cálculo del INJURY SEVERITY SCORE (ISS) en víctimas de trauma cráneo-encefálico. Los resultados mostraron que el valor del ISS coincidía en la mayoría (58,51\%) de las víctimas posibles de codificación por los dos instrumentos. En cuanto a la indicación de la faja de gravedad del trauma (grave, moderado y leve) no existian diferencias estadísticamente significantes entre los dos instrumentos. En cuanto a la capacidad de cobertura de la CAIS/ 85 para la identificación de la gravedad de las lesiones, se constató que la CAIS/85 permitió la puntuación de 61,38\% de las lesiones puntiadas con la AIS/90. 
REFERÊNCIAS BIBLIOGRÁFICAS

01. THE ABBREVIATED Injury Scale: 1985 revision. Des Plaines: Association for the Advancement of Automotive Medicine, 1985.

02. THE ABBREVIATED Injury Scale: 1990 revision. Des Plaines: Association for the Advancement of Automotive Medicine, 1990.

03. ACORN,S.; ROBERTS, E. Head injury: impact on the wives. J. Neurosci. Nurs., v.24, n.6, p.3248, 1992.

04. BERQUÓ, E.S. et al. Bioestatística. São Paulo: EPU,1980.

05. BRASIL. Ministério da Saúde. Secretaria Nacional de Ações Básicas de Saúde. Estatísticas de mortalidade: Brasil, 1989. Brasília: Centro de Documentação, 1993.

06. CAMPBELL,C.H. Needs of relatives and helpfulness of support groups in severe head injury. Rehabil. Nurs., v.13, n.6, p.320-5, 1988.

07. CHAMPION, H.R. et al. The major trauma outcome study: establishing national norms for trauma care. J. Trauma, v.30, n.11, p.1356-65, 1990.

08. CIVIL,I.D.; SCHWAB,C.W. The Abbreviated Injury Scale, 1985 revision: a condensed chart for clinical use. J. Trauma, v.28, n.1, p.87-90, 1988.

09. DALOSSI,T.; KOIZUMI,M.S. Estudo comparativo da gravidade do trauma de pacientes com ou sem traumatismo crânio-encefálico. Rev. Bras. Neurol., v.30, n.6, p.181-9, 1994.

10. DEANE, S.A. et al. The hospital trauma team: a model for trauma management. J.Trauma, v.30, n.7, p. 806-12, 1990.

11. EASTMAN, A.B.; WEST, J.G. Field triage. In: MOORE, E.E. et al. Trauma. 2.ed. California: Appleton-Lange, 1991. cap.5, p.67-79.

12. GARDNER, D. Acute management of the headinjured adult. Nurs.Clin.North Am., v.21, n.4, p.555-62, 1986.

13. GUIRGUIS, E.M. et al. Trauma outcome analysis of two canadian centres using the TRISS method. J. Trauma, v.30, n.4, p.426-9, 1990.
14. JENNETT,B. et al. Prognosis of patients with severe head injury. Neurosurgery, v.4, n.4, p.283-9, 1979.

15. JENNETT, B. et al. Disability after severe head injury: observations on the use of the Glasgow Outcome Scale. J.Neurol. Neurosurg. Psychiatry, v.44, n.4, p.285-93, 1981.

16. KALSBEEK,W.D. et al. The national head and spinal cord injury survey: major findings. J. Neurosurg., v.53, p.S19-31, 1980.

17. KRAUS, J.F. Epidemiology of head injury. In: COOPER, P.R. Head injury. 3.ed. New York: Williams \& Wilkins, 1993. cap.1, p.1-25.

18. LONG,W.B. et al Accuracy and relationship of mecanisms of injury, trauma score, and injury severety score in identifying major trauma. Am.J. Surg., v.151, n.5, p.581-4, 1986.

19. LUERSSEN,T.G. et al. Outcome from head injury related to patient's age: a longitudinal prospective study of adult and pediatric head injury. J.Neurosurg., v.68, p.409-16, 1988.

20. MARSHALL,L.F. et al. The national traumatic coma data bank. J.Neurosurg., v.59, p.276-84, 1983.

21. MASET, A. et al. Epidemiologic features of head injury in Brazil. Arq.Bras.Neurocir., v.12, p.293-302, 1993.

22. RIGGS, J.E. Mortality from accidental falls among the elderly in the United States, 1962-1988: demonstrating the impact of improved trauma management. J. Trauma, v.35, n.2, p.212-9, 1993.

23. SILVA, A.A.D. et al. Traumatismo craniencefálico grave: abordagem inicial e resultados. Arch.Bras.Neurocir., v.8, n.4, p.189-99, 1989.

24. VOLLMER, D.G. Prognosis and outcome of severe head injury.In:COOPER, P.R. Head injury. 3.ed. New York: Williams \& Wilkins, 1993. cap.23, p.553-81.

25. VOLLMER, D.G. et al. Age and outcome following traumatic coma: why do older patients fare worse? J.Neurosurg., v.75, p.S37-49, 1991.

26. YOUNG, B. et al. Early prediction of outcome in head-injured. J.Neurosurg., v.54, p.300-3, 1981. 


\section{ANEXO 1}

Ficha de levantamento de dados do prontuário

Caso ${ }^{\mathrm{o}}$ :

R.G.:

Data de nascimento:

Nome:

Sexo:

Causa externa:

Data do Trauma:

Data da admissão:

Data da alta:

Observações:

Diagnósticos de lesão: 
ANEXO 2

Pontuação das lesões pelo Manual AIS 90², CAIS/85 ${ }^{8}$ e cálculo do ISS

Caso ${ }^{\circ}$ :

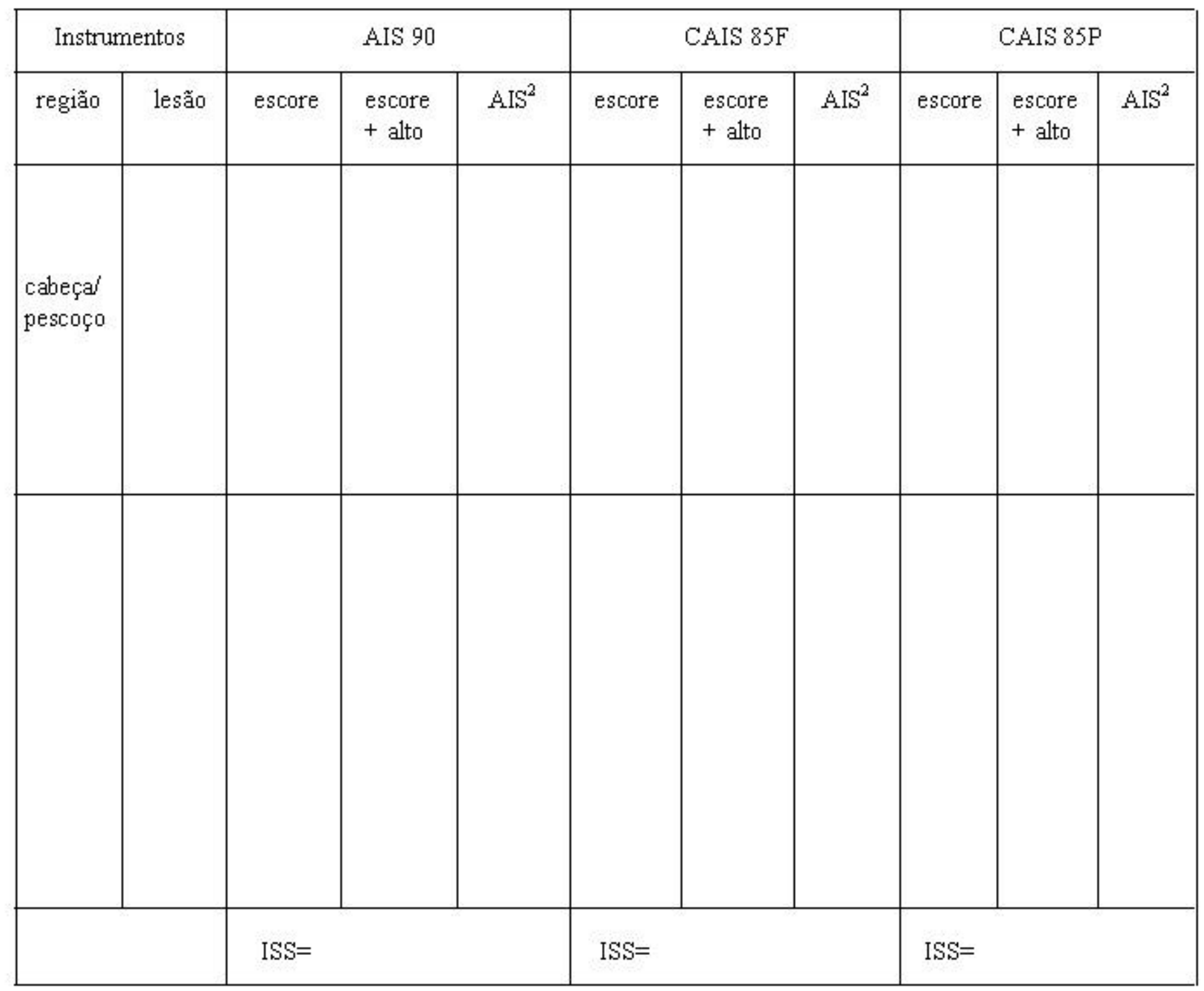

Obs:

Registro: 


\section{ANEXO 3}

\section{Exemplo do cálculo do ISS utilizando-se o Manual AIS/90² e Mapas CAIS/85 ${ }^{8}$}

Caso $n^{\circ}:$

Registro:

\begin{tabular}{|c|c|c|c|c|c|c|c|c|c|c|}
\hline \multicolumn{2}{|c|}{ Instrumentos } & \multicolumn{3}{|c|}{ AIS 90} & \multicolumn{3}{|c|}{ CAIS $85 \mathrm{~F}$} & \multicolumn{3}{|c|}{ CAIS $85 \mathrm{P}$} \\
\hline região & lesão & escore & $\begin{array}{l}\text { escore } \\
+ \text { alto }\end{array}$ & $\mathrm{AIS}^{2}$ & escore & $\begin{array}{l}\text { escore } \\
+ \text { alto }\end{array}$ & $\mathrm{AIS}^{2}$ & escore & $\begin{array}{l}\text { escore } \\
+ \text { alto }\end{array}$ & $\mathrm{AIS}^{2}$ \\
\hline $\begin{array}{l}\text { cabeçae } \\
\text { pescoço }\end{array}$ & $\begin{array}{l}\text { Lesão } \\
\text { axonal } \\
\text { difusa } \\
\text { Hematoma } \\
\text { subdural } \\
\text { Fratura } \\
\text { occipital }\end{array}$ & $\begin{array}{l}4 \\
2\end{array}$ & 5 & 25 & $\begin{array}{l}4 \\
2\end{array}$ & 4 & 16 & \multicolumn{3}{|c|}{$\begin{array}{l}\text { Não aplicável (vítima de } \\
\text { ferimento fechado) }\end{array}$} \\
\hline $\begin{array}{l}\text { membros } \\
\text { e cintura } \\
\text { pélvica }\end{array}$ & $\begin{array}{l}\text { Fratura de } \\
\text { punho } \\
\text { Fratura de } \\
\text { tíbia }\end{array}$ & $\begin{array}{l}2 \\
2\end{array}$ & 2 & 4 & 2 & 2 & 4 & \multicolumn{3}{|c|}{$\begin{array}{l}\text { Não aplicável (vitima de } \\
\text { ferimento fechado) }\end{array}$} \\
\hline & & ISS $=2$ & & & ISS $=20$ & & & ISS $=$ & & \\
\hline
\end{tabular}

Obs:

CALS/85 não possibilita a codificação de lesão axonal difusa 